\title{
A Performance Analysis of Virtual Mail Server on Type-2 Hypervisors
}

\author{
Faizan Ali KHAJI", Sri Varun POTLURI and Anil Kumar KAKELLI \\ Department of Analytics, School of Computer Science \& Engineering, Vellore Institute of Technology, \\ Vellore 632014, India
}

("Corresponding author's e-mail: khajifaizanali123@gmail.com)

Received: 13 April 2020, Revised: 4 May 2021, Accepted: 11 May 2021

\begin{abstract}
Virtualization is now the most efficient and successful procedure to use server resources. Virtualization is a technology that will efficiently allow the instructions or programs based on the available hardware. With the help of virtualization, we can achieve benefits like reduced capital and operating costs, minimized downtime, increased productivity, and good disaster recovery, etc. This work compares the virtual mail server performance on 3 different Hypervisor software. The 3 different type- 2 hypervisor software we chose to compare were Kernel-based Virtual Machine (KVM), Oracle VirtualBox, and VMware. A computer system that sends and also receives an email over a network either on LAN or the internet is called a Mail Server. Although large ISPs and public email services have their dedicated hardware, in most cases, the web servers and mail servers are hosted on a single machine by virtualization. When a Mail server is implemented on the hypervisor, it is then considered as a virtual mail server. A virtual mail server is good at load balancing and has many more benefits. This paper presents how to implement a virtual mail server and shows the comparative results of the virtual mail server on different type-2 hypervisor software.
\end{abstract}

Keywords: Virtualization, Type-2 hypervisors, Performance, Kernel-based Virtual Machine (KVM), Virtual mail server

\section{Introduction}

Virtualization is the way to create a virtual iteration of different computer-related objects like computer hardware platforms, devices used for storage, and computer networking resources. It can also be defined as the distribution of available resources to different applications or platforms with the help of a mainframe known as hypervisors [1]. There are mainly 2 types of virtualizations: full virtualization and para virtualization. In full virtualization, the actual hardware is simulated to allow the operating systems and software to run without any modifications [2]. Whereas in para virtualization, the hardware itself is not simulated, but the apps are run in isolated domains. Few programs have to be modified to run in this type of virtualization [3].

A server that takes care of and delivers e-mail over a network either on LAN or Internet is called a mail server [4]. A mail server can receive emails from the clients and stores them in the server [5]. The mail server can also send the mails to the client computers. A client could be anyone like a personal computer at home or office, a smartphone, etc. A mail program uses an SMTP server to send the mail; it connects to the server with SMTP to send the mail and uses POP3 to download the mail from the server. SMTP and POP3 are the protocols used to connect to the server [6].

When a mail server is hosted on a hypervisor rather than a physical machine, it is called a virtual mail server. The hosting of more than 1 domain on the same hardware system and IP address with overlapping usernames is called virtual mail server hosting. The main advantage of virtual mail servers is 
http://wjst.wu.ac.th

that they are scalable, and they have their dedicated core and memory allocation [7]. They bring down the installation and maintenance costs [8]. They significantly reduce downtime. They can also be completely customized. There are no human users interactively working on the server other than the system administrators [9]. A typical user is working on his device and is accessing the server services. Evaluation results for different criteria confirm the server virtualization technique's high performance and CPU usage and good performance with noticeable agility. Experimental results obtained through our design prototype showed that virtualization of servers can significantly reduce resource consumption while improving system performance. This research work mainly focused on a comparison of a virtual mail server performance on 3 different hypervisor software. The 3 different hypervisor softwares we chose to compare were KVM, Oracle VirtualBox, and VMware.

The paper is structured as follows. Section 2 focuses on the literature survey of virtualisation and challenges around virtual servers. Section 3 presents the operating systems, type- 2 hypervisors, and the implementation of a virtual mail server. Results of performance on the various type-2 hypervisor are discussed in section 4. Finally, in section 5, the conclusion and future work are presented.

\section{Literature survey}

The quality of the present invention provides an electronic mail flow management method, system, and product. In a serial propagation mode, a computing device receives the $1^{\text {st }}$ electronic mail from a sender addressed to the $1^{\text {st }}$ and $2^{\text {nd }}$ recipients, and in response, the computing device transmits the $1^{\text {st }}$ electronic mail enroot to the $1^{\text {st }}$ recipient, but not the $2^{\text {nd }}$. In response to the $1^{\text {st }} e$-mail, a computer device receives a $2^{\text {nd }} \mathrm{e}$-mail from the $1^{\text {st }}$ recipient and a computer device transmits the $1^{\text {st }}$ e-mail to the $2^{\text {nd }}$ recipient [10]. The present innovation is generally concerned with electronic mail processing and, more precisely [11], with electronic mail servers that monitor the dissemination of an email to addresses based on a sender-determined criterion as shown in Table 1.

A server management method and system are disclosed to allow a user to control a server scheduling via a remote terminal device. To receive and execute management commands, a management program module is installed in the server [12]. The program management module transmits a management order to the server's operating and/or control mechanism based on management information sent by the user from the remote terminal machine and via a network communication system. According to the received management command, the operating and/or control mechanism can control and manage the server. The above process and system of server management allows the user to manage and use the server remotely, thereby increasing operating efficiency.

A virtual email server system provides an incoming message server with at least 1 PDA to receive an email. The mail agent sends a signal to the preprocess agent to query identity information for the last message after a predetermined time interval [13]. Then the mail agent checks the incoming message server according to identity information and receives a new message from the incoming message server. The mail agent then forwards the new message to the content converter agent to transform the new message's content into an internally formatted message. Finally, the content converter agent sends the message formatted internally to the preprocess agent, and the preprocess agent stores the message formatted internally to the database.

VMware ESX Server is a hardware resource multiplexer i.e., a virtual machine software [14]. The performance of an ESX Server architecture key component: its storage subsystem. We perform a chain of benchmarks on various storage systems to conclude the performance of native systems and virtual machines. We aim to show that the performance of the virtual machine and the native machine are similar and also, they behave in the same manner. A mail server will maintain a password conversion table that shows a relation between the registered user, a formal password, and a virtual password which varies from the formal password [15]. The user has essentially notified a password. When the client computer sends the virtual password to the business intruder, the conversion processor transforms it to the formal password from the virtual password, and the formally passworded mail is transmitted by the task processor of the SMTP system to the general-purpose mail server. A computer device customer cannot enter the general mail server by virtual password [15]. 
Table 1 The comparative analysis of the existing techniques.

\begin{tabular}{|c|c|c|}
\hline S.no & Name of the technique & Challenges in the existing system \\
\hline 1. & $\begin{array}{l}\text { File system performance comparison in } \\
\text { full hardware virtualization with ESXi } \\
\text { and Xen hypervisors [21] }\end{array}$ & $\begin{array}{l}\text { Performance comparison is only on a file system. It is limited } \\
\text { to type- } 1 \text { /full hardware hypervisors only. }\end{array}$ \\
\hline 2. & $\begin{array}{l}\text { Performance Comparison of the type-2 } \\
\text { hypervisor VirtualBox and VMware } \\
\text { Workstation [18] }\end{array}$ & $\begin{array}{l}\text { Comparison only between } 2 \text { type- } 2 \text { hypervisors. Performance } \\
\text { is limited to the applied file system. }\end{array}$ \\
\hline 3. & $\begin{array}{l}\text { Competition of virtualized ext } 4 \text {, xfs and } \\
\text { btrfs filesystems under the type- } 2 \\
\text { hypervisor [22] }\end{array}$ & $\begin{array}{l}\text { Performance analysis on } 3 \text { different file systems. Performance } \\
\text { analysis is limited to } 1 \text { type- } 2 \text { hypervisor. The focus is on } \\
\text { read and write throughputs calculated under various workload } \\
\text { conditions. }\end{array}$ \\
\hline 4. & $\begin{array}{l}\text { Performance analysis of virtualized } \\
\text { embedded computing systems [23] }\end{array}$ & $\begin{array}{l}\text { The work provides a concise overview of } 3 \text { common } \\
\text { virtualization methods for embedded systems. The work is } \\
\text { limited to the analysis of embedded systems. Only QEMU is } \\
\text { taken under consideration for analysis. }\end{array}$ \\
\hline 5. & $\begin{array}{lcr}\text { Comparing } & \text { VMware Fusion, } & \text { Oracle } \\
\text { VirtualBox, } & \text { Parallels } & \text { Desktop } \\
\text { implemented as type-2 hypervisors [24] }\end{array}$ & $\begin{array}{l}\text { The work compared and benchmarked } 3 \text { dominant leading } \\
\text { type- } 2 \text { hypervisors (Parallels Desktop, Oracle VirtualBox, } \\
\text { and VMware Fusion) that offer processor virtualization. This } \\
\text { work has focused on many benchmarks. Network throughput } \\
\text { is compared but does not concentrate on the mail server. }\end{array}$ \\
\hline
\end{tabular}

\section{Materials and methods}

For this investigation, the analysis was carried on Ubuntu 18.02 LTS as the host machine and Windows 10 pro (Version: 1709) [Build: 16299.98] as our guest machine. Likewise, the 3 virtualization hypervisor software used to compare were Oracle VirtualBox, VMware, and KVM.

\section{Ubuntu}

Ubuntu is an open source software that can be downloaded anywhere from Debian. It is available on 3 platforms: desktops, servers, and cores. All the versions can be used over the computer alone or a virtual machine. Ubuntu is very popular for cloud computing because it gives support for OpenStack [16].

\section{Windows}

In response to the growing attraction towards GUIs, Windows is the graphical operating system shell for MS-DOS rebased by Microsoft. Windows now occupy over $90 \%$ of the personal computer market overtaking the Mac OS by Apple.

\section{Oracle VirtualBox}

VirtualBox is a free platform for virtualization. It can be installed on existing operating systems like Windows, Mac, and Linux. It is quick, but very powerful because it can run on a small integrated device, desktops, even data centers, and cloud environments [17]. On a large number of 32-bit and 64-bit operating systems, VirtualBox runs. This needs an operating system built to run parallel to existing applications on the host. On all platform hosts, VirtualBox is practically similar when using the same image formats and filesystem. The virtual machine built on the host computer can be run on another platform, even though there are different operating systems globally. 
http://wjst.wu.ac.th

\section{VMware Player}

VMware Player is a free VMware, Inc. program for virtualization. It can be used to work with existing virtual machines along with those created on its own. Its core is similar to the VMware Workstation which is a software with more capabilities but commercially available [4]. The VM player lacks a few features like backups, clones, virtual rights management that are available in a workstation. Besides the VMware player software, it uses a few drivers to improve pace and provide more functionality any other versions of VMware will support the virtual machines developed by another version of VMware. In addition, with the aid of the VMware vCenter Converter, we can deploy any virtual machines produced from 1 manufacturer and hardware from other manufacturers either by converting or directly [18].

\section{KVM}

KVM is a free platform for virtualization based on the Linux kernel [19]. It is in kernel version 2.6.20, which was released on February 5, 2007, into the Linux kernel mainline. KVM requires a virtualization hardware extension processor like Intel VT or AMD-V. KVM has also been transported in the form of loadable kernel modules to other operating systems such as FreeBSD and illumos. In addition, KVM offers para-virtualization support to visitors using the VirtIO API for Linux, OpenBSD, FreeBSD, NetBSD, Plan 9, and Windows [20]. It includes a paravirtual Ethernet chip, disk I/O controller, balloon module, and SPICE or VMware driver VGA graphics adapter.

\section{Virtual mail server}

The work aims to determine the performance of virtual mail servers on the different type-2 hypervisor. The different type-2 hypervisor software was hosted on the Ubuntu 18.02 LTS operating system. The work provides the conclusion that the observations were compared with the various sizes of data transmitted on the local area network using virtual mail servers. All experiments were carried out on the same hardware and disk partition to preserve reliability and produce accurate results. Windows 10 pro was used as a guest operating system in KVM, Oracle VirtualBox, and VMware. To gain the impact of the virtualization, the virtual mail server was hosted on the virtual machine as shown in Figure 1. 
http://wjst.wu.ac.th

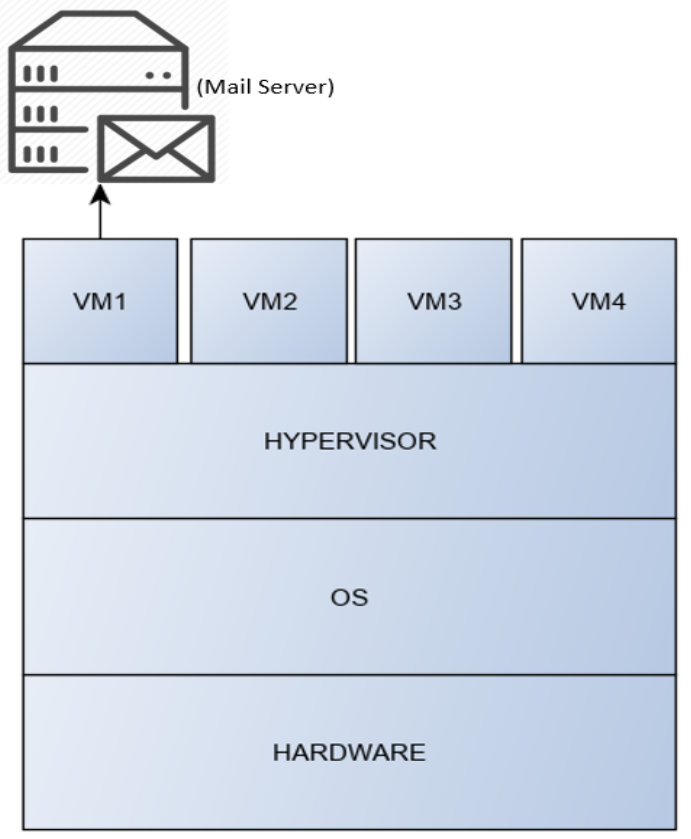

Figure 1 Block diagram of Virtual Mail server.

As virtualization has many benefits, virtual mail server also gives similar benefits to the users. With virtual mail server speedy recovery time, better scalability, cost savings, and many more can be achieved. A virtual machine (VM) is a software that functions similarly to the actual machine [1]. The VMM (Virtual Machine Manager) is a device that can provide organizational oversight over multiple virtual machines on which different operating systems can be established. These deployed operating systems will get the messages from the application running over them and virtual machines will handle the hardware operation by hardware capabilities which are sent by the mounted operating systems.

There are mainly 2 types of hypervisors: (1) Type-1 hypervisor is directly implemented on the hardware, so it reaches better performance. (2) Type-2 hypervisor will be hosted by a guest operating system so, it delivers a low level of performance compared to Type (1) hypervisor [3]. Type-1 hypervisor is an example of kernel class, it is also called bare-metal hypervisor because it works directly on the hardware on which its operating system is mounted. Different type-1 hypervisors software's are Xen, Hyper-V, ESX Server, etc. Among Type-1 hypervisors, KVM is the most influential [7]. Type-2 hypervisor runs on an operating system, and partially 1 system application. Guest operating systems are mounted just above the hypervisor. Different type-2 hypervisor software's are VMware Workstation, Oracle VirtualBox, QEMU KVM, Microsoft's virtual CPU, etc. [3].

\section{Methodology and implementation}

A mail server was hosted on type-2 hypervisors. This mail server was set up and configured to run efficiently. Mails of different sizes were sent and received over this new mail server. To compare the performance, logs of mail servers hosted on different type-2 hypervisors were analyzed with required hardware and software are presented in Table 2. Based on the analysis, the mail server with the best environment could be detected using Figure 2. 
http://wjst.wu.ac.th

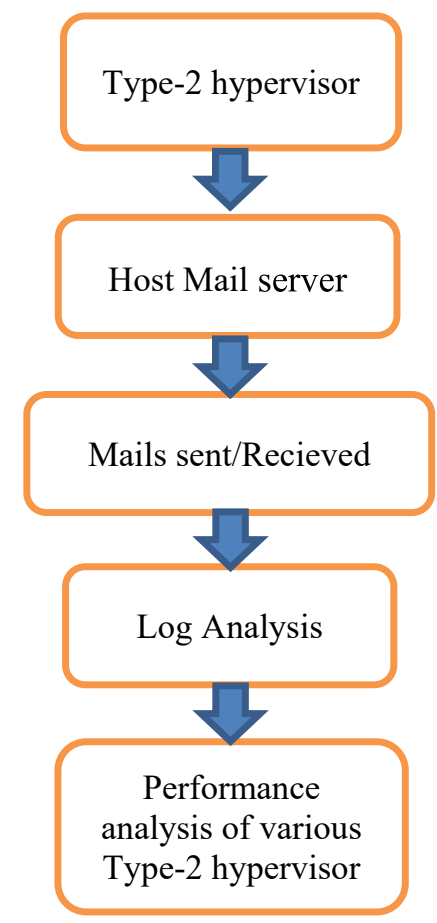

Figure 2 Methodology for performance analysis.

Mail server software named hMailServer is a free open-source email server. It was hosted over 3 different type-2 hypervisors. Various mail ids were registered over these mail servers to send/receive emails. Using Outlook, emails were sent/received over different mail ids. Mails of 3 different sizes (1, 5, and $10 \mathrm{~Kb}$ ) were sent over the local area network using this mail server. Logs of each mail server were compared and analyzed to know the best type- 2 hypervisor for a virtual mail server.

\section{Results and discussion}

Evaluation 1

Evaluation 1 includes a transfer of $1 \mathrm{~Kb}$ of email data on different type-2 hypervisor software. On each type- 2 hypervisor software, 5 emails were sent on a local area network and the average of time taken to send emails from sender to receiver was calculated. Figure 3 displays the evaluation outcomes for this particular case. 
$1 \mathrm{~Kb}$

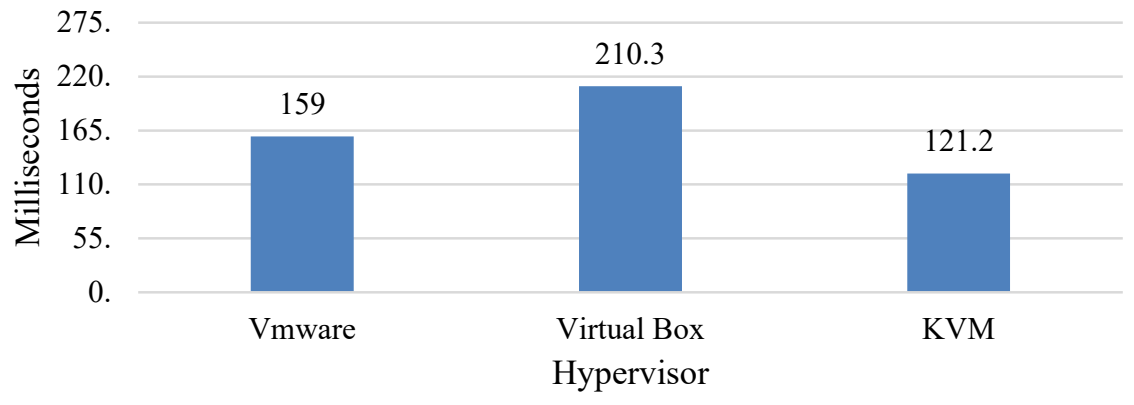

Figure 3 The average time to send $1 \mathrm{~Kb}$ of email data on different virtual mail servers.

Table 2 Specifications used for the experimentation.

\begin{tabular}{cc}
\hline Equipment & Specifications \\
\hline Hard disk & $\begin{array}{c}\text { Toshiba mq01abd100, 1TB of capacity, 5400rpm speed, } \\
\text { SATA Interface. }\end{array}$ \\
\hline Hp laptop & $\begin{array}{c}\text { Intel Core i5-6200 CPU @2.30 GHZx4, Windows 10 } \\
\text { operating system/Ubuntu 18.02Lts (Dual Boot), } \\
\text { Memory:7.7GiB }\end{array}$ \\
\hline $\begin{array}{c}\text { Realtek rt18723be } 802.11 \mathrm{~b} / \mathrm{g} / \mathrm{n} \text { WIFI } \\
\text { adapter }\end{array}$ & Frequency band: 2.4 GHz \\
\hline Guest operating system & Windows 10 pro 64-bit \\
\hline vCPUs & Virtual Environment \\
\hline Hard disk & each processor has 2 cores with a total of 4 cores \\
\hline
\end{tabular}

In evaluation 1, KVM performance is approximately $40 \%$ better than VirtualBox and VMware performance is approximately $25 \%$ better than VirtualBox. This dominant advantage has been gained by KVM.

\section{Evaluation 2}

$3 \mathrm{~Kb}$ of email data was transferred on different type-2 hypervisor software. The size of email data was increased 3 times to check for any abnormalities in performance. The average time taken for sending emails was calculated on each hypervisor for comparison of performance. Figure 4 displays the evaluation outcomes for this particular case.

In evaluation 2, the performance of KVM is about $40 \%$ higher than that of VirtualBox and VMware. The time taken to send emails by VMware had a rapid change with a rise of $55 \mathrm{~ms}$ with an increase in $2 \mathrm{~Kb}$ of data. Even in this evaluation, the best performance of KVM remains the same. 
http://wjst.wu.ac.th

\section{$3 \mathrm{~Kb}$}

260.

247

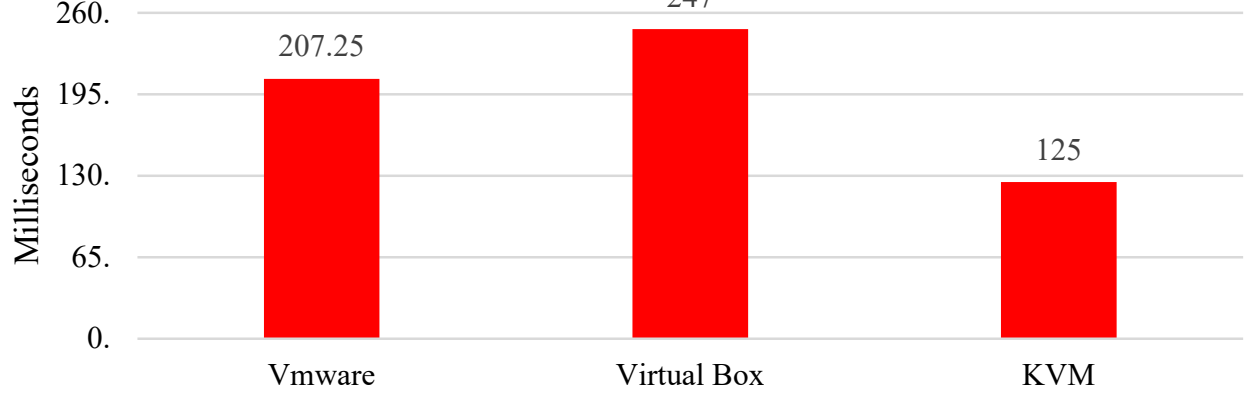

Hypervisor softwares

Figure 4 Average time to send $3 \mathrm{~Kb}$ of email data on different virtual mail servers.

\section{Evaluation 3}

On different type-2 hypervisor software email data of size about $10 \mathrm{~Kb}$ was transferred. On each hypervisor, the average time taken to send emails was calculated for comparison. Figure $\mathbf{5}$ displays the evaluation outcomes for this particular case.

$10 \mathrm{~Kb}$

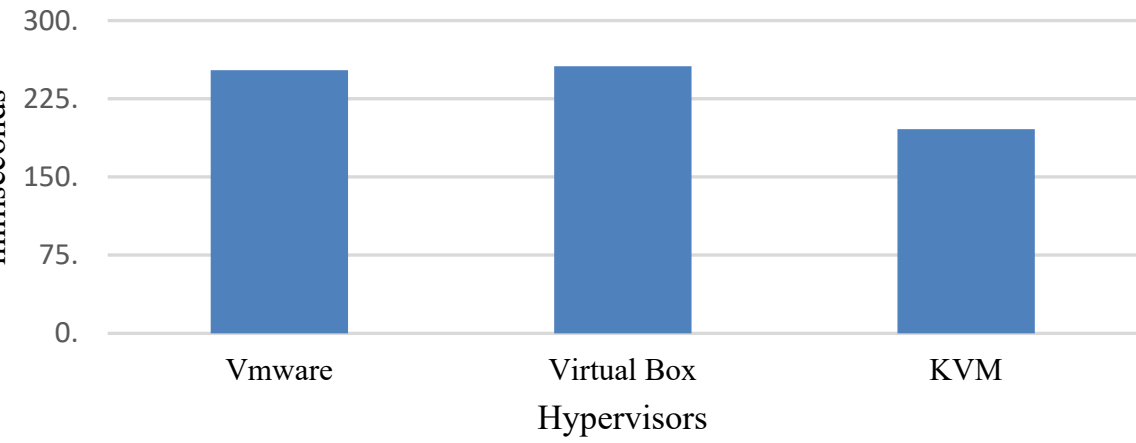

Figure 5 Average time to send $10 \mathrm{~Kb}$ of email data on different virtual mail servers.

In evaluation 3, KVM performance is approximately $30 \%$ better than VirtualBox and VMware. The performance of VirtualBox and VMware were close but VMware performance was good when compared to VirtualBox. Even with an increase in data size KVM was performing well for a virtual mail server. 


\section{Conclusions and future work}

The virtual mail server has greater advantages than the normal mail server. Faster redeploy of mail server can be achieved in minutes and backups of mail server can be achieved using virtualization whereas physical mail servers cannot be achieved easily. The amount of hardware used decreases with virtual mail servers which drastically decreases cost. Testing becomes easier using virtual mail servers as previous snapshots can be reverted easily even if there is any huge loss of data in the server during testing. Disaster recovery is achieved quickly which boosts the usage of virtual mail servers in a company.

The experiments were performed on the same hardware and software so that the evaluation integrity of different type-2 hypervisor does not decrease. Evaluations were performed with different data sizes so that the quality of comparing type- 2 hypervisor software increases. Based on the evaluation results obtained, it can be inferred that the KVM software performs best for small to huge size data, usually $1 \mathrm{~B}$ $10 \mathrm{~Kb}$.

The mail server with a huge number of clients can be deployed on a type-1 hypervisor to achieve the best results. Different type-1 hypervisor software can be compared to the best software for use as a virtual mail server. Paravirtualization can also be used for better results in terms of handling huge number of clients.

\section{References}

[1] N Jain and S Choudhary. Overview of virtualization in cloud computing. In: Proceedings of the Symposium on Colossal Data Analysis and Networking, Indore, India. 2016, p. 1-4.

[2] F Yile. Utilizing the virtualization technology in computer operating system teaching. In: Proceedings of the $8^{\text {th }}$ International Conference on Measuring Technology and Mechatronics Automation, Macau, China. 2016, p. 885-8.

[3] İ Arslan and İG Özbilgin. Virtualization and security: Examination of a virtualization platform structure. In: Proceedings of the International Conference on Computer Science and Engineering, Antalya, Turkey. 2017, p. 221-6.

[4] Z Wua, J Tang, CK Kwong and F Marinelli. An improvement on Integrated production strategy and reuse scenario: A CoFAQ model and case study of mail server system development. Omega 2015; 56, 50-2.

[5] Z Davitadze, G Kakhiani and G Beria. Remote administration of information systems via e-mail. In: Proceedings of the IEEE East-West Design \& Test Symposium, Batumi, Georgia. 2019, p. 1-5.

[6] P Menegay, J Salyers and G College. Secure communications using blockchain technology. In: Proceedings of the IEEE Military Communications Conference, Los Angeles, CA, USA. 2018, p. 599-604.

[7] Y Chen, I Liu, C Chou, J Li and C Liu. Multiple virtual machines live migration scheduling method study on VMware vMotion. In: Proceedings of the $3^{\text {rd }}$ International Conference on Computer and Communication Systems, Nagoya, Japan. 2018, p. 113-6.

[8] F Liu, Z Ma, B Wang and W Lin. A virtual machine consolidation algorithm based on Ant Colony system and extreme learning machine for cloud Data Center. IEEE Access 2020; 8, 53-67.

[9] AS Abohamama and E Hamouda. A hybrid energy-Aware virtual machine placement algorithm for cloud environments. Expert Syst. Appl. 2020; 150, 113306.

[10] E Russell and SA Woods. Personality differences as predictors of action-goal relationships in workemail activity. Comput. Hum. Behav. 2020; 103, 67-79.

[11] MZ Sabir and M Yousaf. Design and implementation of an end-to-end web based trusted email system. Procedia Comput. Sci. 2018; 141, 231-8.

[12] R Cura, J Perret and N Paparoditis. A scalable and multi-purpose point cloud server (PCS) for easier and faster point cloud data management and processing. ISPRS J. Photogramm. Remote Sens. 2017; 127, 39-56. 
http://wjst.wu.ac.th

[13] A Bhardwaj and S Goundar. Security challenges for cloud-based email infrastructure. Netw. Secur. 2017; 11, 8-15.

[14] R Mohtasin, PWC Prasad, A Alsadoon, G Zajko, A Elchouemi and AK Singh. Development of a virtualized networking lab using GNS3 and VMware workstation. In: Proceedings of the International Conference on Wireless Communications, Signal Processing and Networking, Chennai, India. 2016, p. 603-9.

[15] Z Davitadze, G Kakhiani and G Beria. Remote administration of information systems via e-mail. In: Proceedings of the IEEE East-West Design \& Test Symposium, Batumi, Georgia. 2019, p. 1-5.

[16] X Ren, Q Huang, X Xia, Z Xing, L Bao and D Lo. Characterizing common and domain-specific package bugs: A case study on Ubuntu. In: Proceedings of the IEEE $42^{\text {nd }}$ Annual Computer Software and Applications Conference, Tokyo, Japan. 2018, p. 426-31.

[17] J Bao, X Zhang, D Fan and W Gong. Research on virtual box-based small-scale rural medicalexpert-system. In: Proceedings of the International Conference on Biomedical Engineering and Computer Science, Wuhan, China. 2010, p. 1-4.

[18] DT Vojnak, BS Đorđević, VV Timčenko and SM Štrbac. Performance comparison of the type-2 hypervisor VirtualBox and VMware workstation. In: Proceedings of the $27^{\text {th }}$ Telecommunications Forum, Belgrade, Serbia. 2019, p. 1-4.

[19] C Guo, T Li, Z Gong and H Han. A virtual vulnerability validation platform based on KVM. In: Proceedings of the IEEE $5^{\text {th }}$ International Conference on Electronics Information and Emergency Communication, Beijing, China. 2015, p. 228-31.

[20] R Dantas, D Sadok, C Flinta and A Johnsson. KVM virtualization impact on active round-trip time measurements. In: Proceedings of the IFIP/IEEE International Symposium on Integrated Network Management, Ottawa, ON, Canada. 2015, p. 810-3.

[21] B Đorđević, V Timčenko, N Kraljević and N Davidović. File system performance comparison in full hardware virtualization with ESXi and Xen hypervisors. In: Proceedings of the $18^{\text {th }}$ International Symposium INFOTEH-JAHORINA, East Sarajevo, Bosnia and Herzegovina. 2019, p. $1-5$.

[22] D Pesic, B Djordjevic and V Timcenko. Competition of virtualized ext4, xfs and btrfs filesystems under type-2 hypervisor. In: Proceedings of the $24^{\text {th }}$ Telecommunications Forum, Belgrade, Serbia. 2016, p. 1-4.

[23] D Mathew and BA Jose. Performance analysis of virtualized embedded computing systems. In: Proceedings of the $7^{\text {th }}$ International Symposium on Embedded Computing and System Design, Durgapur, India. 2017, p. 1-5.

[24] R Pandey. Comparing VMware fusion, Oracle VirtualBox, parallels desktop implemented as type-2 hypervisors. 2020. 